\title{
Redox-dependent Structure and Dynamics of Macrophage Migration Inhibitory Factor Reveal Sites of Latent Allostery
}

Erin Skeens, ${ }^{1}$ Meagan Gadzuk-Shea, ${ }^{2}$ Dilip Shah, ${ }^{3}$ Vineet Bhandari, ${ }^{3}$ Devin K. Schweppe,${ }^{2}$ Rebecca B. Berlow, ${ }^{4 *}$ and George P. Lisi ${ }^{1 *}$

${ }^{1}$ Department of Molecular Biology, Cell Biology, \& Biochemistry, Brown University, Providence, RI 02903

${ }^{2}$ Department of Genome Sciences, University of Washington, Seattle, WA 98195

${ }^{3}$ Section of Neonatology, Department of Pediatrics, Cooper University Hospital, Camden, NJ 08103

${ }^{4}$ Department of Integrative Structural and Computational Biology, The Scripps Research Institute, La Jolla, CA 92037

*To whom correspondence should be addressed: George P. Lisi (george_lisi@brown.edu)

Rebecca B. Berlow (rberlow@scripps.edu)

ORCID: Erin Skeens (0000-0002-0580-1291)

Rebecca B. Berlow (0000-0003-1934-0139)

George P. Lisi (0000-0001-8878-5655)

Meagan Gadzuk-Shea (0000-0001-9496-529X) 


\section{Abstract}

Macrophage migration inhibitory factor (MIF) is a multifunctional immunoregulatory protein that is a key player in the innate immune response. Given its overexpression at sites of

4 inflammation in a wide range of diseases marked by increasingly oxidative cellular environment, a comprehensive structural understanding of how cellular redox conditions may impact the

6 structure and function of MIF is necessary. We used solution NMR spectroscopy and mass spectrometry to investigate structural and dynamic signatures of MIF under varied solution redox conditions. Our results indicate that the MIF structure is modified and becomes increasingly dynamic in an oxidative environment, which may be a means to alter the MIF functional response in a redox-dependent manner. We identified latent allosteric sites within MIF that are redox-

11 sensitive and mutational analysis reveals that loss of redox-responsive residues attenuates

12 activation of the coreceptor CD74. Leveraging sites of redox-sensitivity therefore reveals an 13 avenue to modulate MIF function in its "disease state" via structure-based drug design.

Introduction

Macrophage migration inhibitory factor (MIF) is a ubiquitous human protein that plays a crucial role in the pathophysiology of inflammation. ${ }^{1}$ MIF is expressed by nearly every cell in the body ${ }^{1}$ and many of its effects are elicited by its binding to the chemokine receptors CXCR2 and CXCR4 ${ }^{2,3}$ or the coreceptor CD74. ${ }^{4-6}$ Circulating levels are reported in the low $\mu \mathrm{M}$ range in

20 healthy humans, ${ }^{7-9}$ but MIF is overexpressed under inflammatory conditions and has been linked

21 to glucocorticoid overriding activity, ${ }^{10}$ asthma, ${ }^{7,11}$ rheumatoid arthritis, ${ }^{12}$ colitis, ${ }^{13}$ pancreatitis,${ }^{14}$

22 acute respiratory distress syndrome (ARDS), ${ }^{15}$ and cancer ${ }^{16-20}$ as well as complications from

23 COVID-19. ${ }^{21}$ Efforts to elucidate the pathogenic mechanism of MIF have attempted to connect 
24 its disease state to cellular redox properties, ${ }^{11}$ most recently by investigating its cysteine

25 residues as conformational switches. ${ }^{22}$ Mutation of either C56 or C59 in a canonical ${ }^{56} \mathrm{CXXC}{ }^{59}$

$26\left(\mathrm{XX}=\mathrm{AL}\right.$ in MIF) motif modulates the enzymatic and macrophage activation functions of MIF ${ }^{23,24}$

acknowledged contribution of redox imbalance to asthma, ${ }^{26,27}$ ARDS,${ }^{28}$ pulmonary fibrosis, ${ }^{29}$ and

30 cancer, ${ }^{30}$ as well as the link between these pathologies and MIF, very little structural work has

31 directly addressed the redox behavior of MIF. We wondered whether MIF could utilize redox

32 imbalances to act as a pro-inflammatory sensor to toggle its structure based on the chemical

33 environment of the cell. Altered conformations of MIF at local sites of inflammation could then

34 modulate its function or create different modes of interaction with accessory proteins, thereby

35 controlling downstream biological responses. In a first step toward exploring such a mechanism,

36 we investigated the impact of solution redox conditions on the MIF structure and identified

37 regions of the protein that are sensitive to oxidative environments. We used solution nuclear

38 magnetic resonance (NMR) spectroscopy to assess the redox-dependent dynamics of MIF with

39 spin relaxation experiments and used mass spectrometry to identify redox-modified cysteine

40 residues. We then leveraged these findings, using further NMR structural studies and an in vivo

41 assay, to confirm redox-sensitive amino acids as latent points of functional control in MIF.

43 Results

44 Oxidizing solution alters MIF structural stability

45 To assess the effect of solution redox potentials on the MIF structure, we performed far46 UV circular dichroism experiments on redox-neutral MIF samples as well as MIF samples that 
47 had been oxidized or reduced. CD spectra of redox-altered MIF show characteristic $\alpha-\beta$

48 structure, with only minor differences (Figure S1A). These data are consistent with numerous

49 MIF structures in the Protein Data Bank (PDB) that have no obvious alterations in the presence

50 of substrates, inhibitors, or mutations. Despite similarly folded structures, temperature-

51 dependent CD experiments (Figure S1B) show that an oxidizing environment destabilizes MIF

52 to unfolding relative to reduced and redox-neutral MIF $\left(\Delta T_{\mathrm{m}}=-4.56{ }^{\circ} \mathrm{C}, \Delta \Delta \mathrm{G}=-2.65 \mathrm{kcal} / \mathrm{mol}\right.$,

53 Figure S1C). This energetic difference (expressed per trimer) is modest, but suggests a

54 loosening of the MIF structure that may affect its conformational ensemble.

56 Redox-dependent NMR spectra of MIF highlight multiple conformations and altered dynamics

We used solution NMR to pinpoint regions of the MIF structure that are sensitive to the

58 redox environment. ${ }^{1} \mathrm{H}^{15} \mathrm{~N}$ TROSY-HSQC spectral overlays of reduced, oxidized, and redox-

neutral MIF (Figure S2) show that while reduced and redox-neutral samples are spectrally

60 similar, more significant spectral changes are observed for MIF under oxidizing conditions.

61 Redox-sensitive chemical shift perturbations (relative to redox-neutral MIF) are observed

62 throughout the protein, including for residues at the $\mathrm{N}$-terminal enzymatic active site, solvent

63 channel, and monomer-monomer interface (Figures 1A). Additionally, for oxidized MIF samples,

64 duplicate resonances are observed for 22 residues (Figures 1B, S3), suggesting that an

65 oxidizing environment modulates an equilibrium between two conformations of MIF on the NMR

66 timescale. Sites of slow exchange localize to the monomer-monomer interface (Figure 1C),

67 indicating that the structure of this region of the protein is strongly affected by oxidizing solution,

68 consistent with the lower thermal stability observed by CD. Perturbations to MIF are not exclusive

69 to redox-active Cys residues, at times occurring $10-20 \AA$ from the ${ }^{56} \mathrm{CALC}{ }^{59}$ motif or C80. 
70 However, Cys and Met residues serve as nucleation points for our observations, with a majority

71 of redox-dependent changes to the MIF structure occurring proximal to these traditionally

72 sensitive sites (Figure S4). These data are consistent with an alternate oxidized MIF

73 conformation, "oxMIF," most recently probed in a synthetic peptide comprising the MIF ${ }^{56}$ CALC ${ }^{59}$

74 sequence. $^{22}$

75
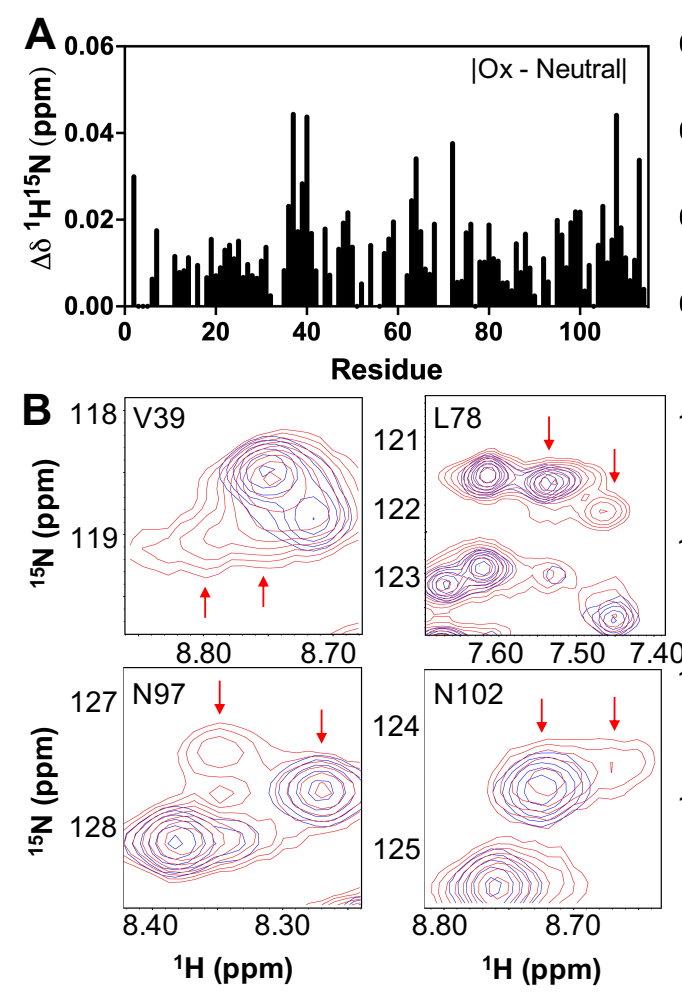
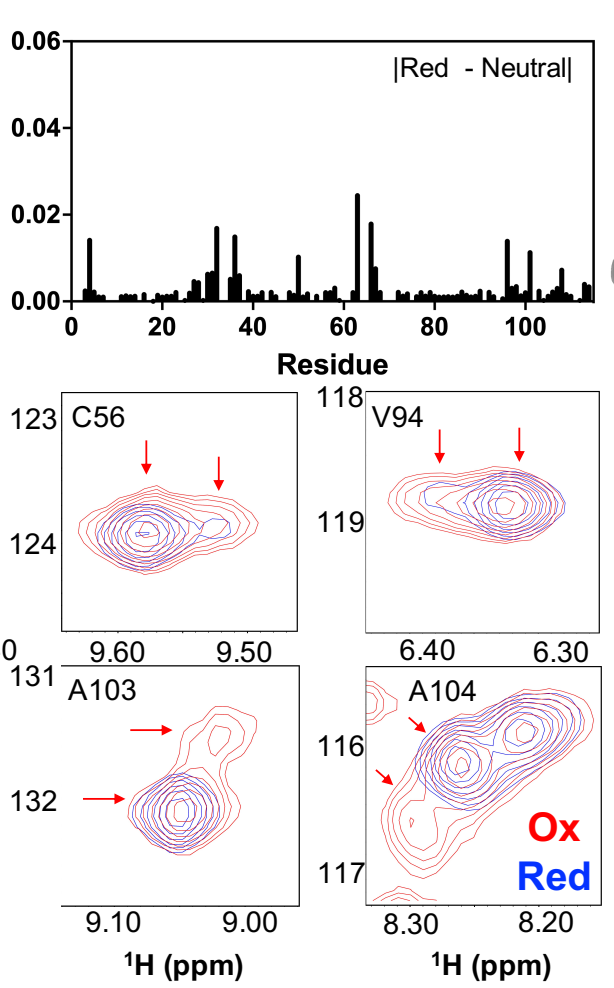

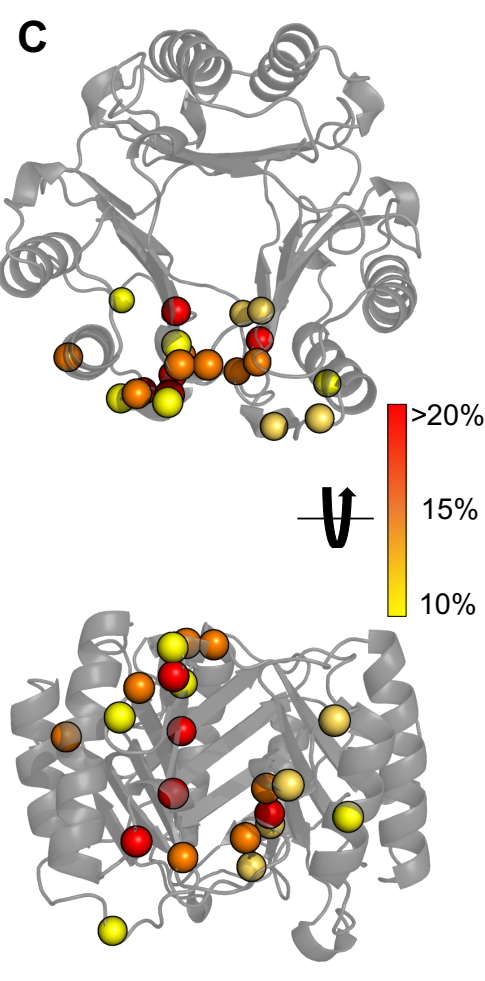

Figure 1. Redox-dependent changes in MIF detected by NMR. (A) ${ }^{1} \mathrm{H}^{15} \mathrm{~N}$ combined chemical shift perturbations $(\Delta \delta)$ for oxidized and reduced MIF, relative to a redox-neutral sample. (B) Selected resonances from ${ }^{1} \mathrm{H}^{15} \mathrm{~N}$ HSQC spectral overlays of reduced (blue) and oxidized (red) MIF. Sites of slow exchange observed under oxidizing conditions are indicated by arrows. Populations of MIF conformers determined from the volumes of slow exchange resonances are mapped onto the MIF trimer (PDB: 1MIF) in (C), where the percentage indicated in the legend denotes the minor species (i.e. satellite peak). Volumes and populations of slow exchange resonances can be found in Figure S3.

Analysis of longitudinal $\left(R_{1}\right)$ and transverse $\left(R_{2}\right)$ NMR relaxation rates ${ }^{31}$ highlights local differences caused by redox conditions. Consistent with the altered thermodynamic parameters of the oxidized MIF samples, individual relaxation rates, as well as the $R_{1} R_{2}$ product, qualitatively 
88 imply that MIF exhibits heightened flexibility under oxidizing conditions (Figure S5A). Analysis

89 of $R_{1} R_{2}$ values ${ }^{31}$ highlights 21 sites suggestive of $\mu \mathrm{s}-\mathrm{ms}$ flexibility in oxMIF $\left(R_{1} R_{2}>1.5 \sigma\right.$ above

90 the $10 \%$ trimmed mean of the data), 7 of which do not appear flexible in both redox-neutral and

91 reduced MIF. Despite local patches of altered flexibility, the dynamic profiles of reduced and

92 redox-neutral MIF are similar to each other. ${ }^{1} \mathrm{H}-\left[{ }^{15} \mathrm{~N}\right]$ NOE values and order parameters also hint

93 at patches of local flexibility in oxMIF, though fast timescale dynamics do not differ substantially

94 between samples. Residues with relaxation parameters suggestive of $\mu s$ - ms flexibility are

95 mapped onto the MIF trimer and monomer structures in Figure S5. Correlation plots of $R_{1} R_{2}$

96 and $S^{2}$ parameters for reduced and oxidized MIF reveal a weaker correlation for $R_{1} R_{2}$,

97 suggesting that MIF experiences greater dynamic changes on the $\mu \mathrm{s}-\mathrm{ms}$ timescale over a

98 range of redox conditions (Figure $2 \mathrm{~A}$ ). Contributions to the variations in $R_{1} R_{2}$ parameters are

99 most strongly affected by oxidizing solution conditions (Figure 2B, left panel), while reducing

100 conditions contribute more significantly to the ps - ns dynamics of MIF via $S^{2}$ (Figure 2B, right

101 panel). Redox-sensitive residues with apparent $\mu \mathrm{s}$ - ms flexibility include T30, G50, L58, S63,

$102 \mathrm{I64}{ }^{*}, \mathrm{~K} 66, \mathrm{R} 73, \mathrm{~L} 83, \mathrm{~A} 84, \mathrm{R} 86, \mathrm{R} 93, \mathrm{I96}, \mathrm{N} 97^{*}, \mathrm{~N} 109^{*}, \mathrm{~N} 110, \mathrm{~T} 112$, and A114, and those with 103 apparent ps - ns fluctuations are F3, T7, L26, T30, D44, I64*, K66, K77, L83, L87, S90, Y95*, 104 D100, A103, and S111* (Figure 3C). Asterisks indicate residues previously identified as 105 controlling MIF enzymatic activity and CD74 activation via direct interaction or through allosteric 106 signaling, ${ }^{6,32,33}$ indicating redox-dependent perturbations in MIF may have functional 107 consequences. 

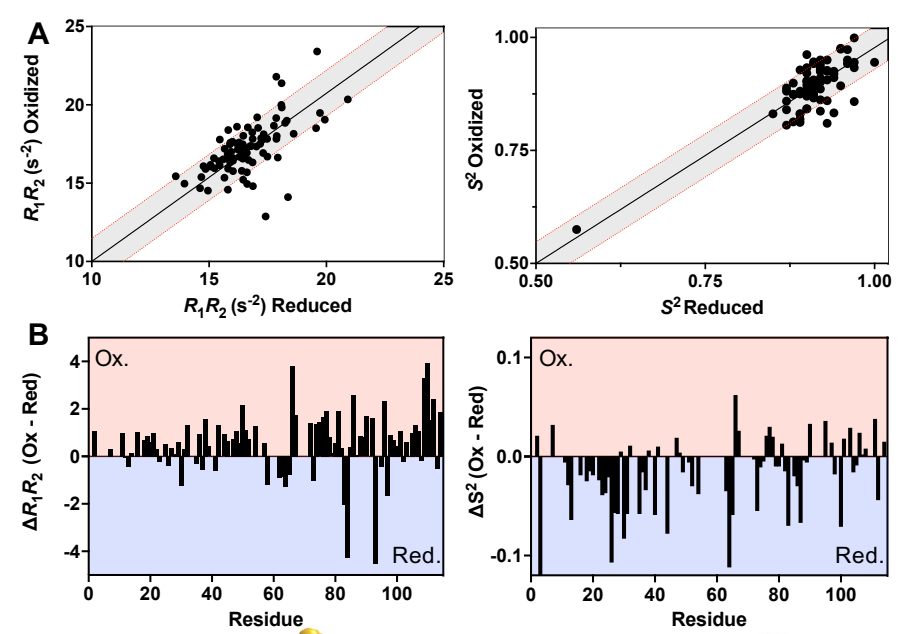

108

C

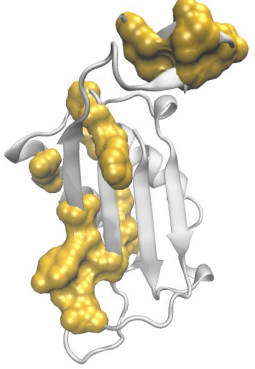

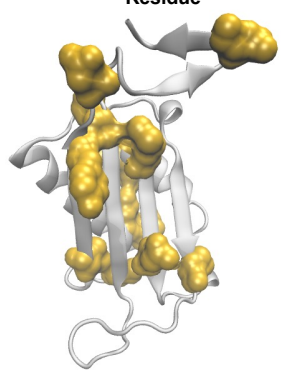

Figure 2. (A) Correlation plots of the $R_{1} R_{2}$ (left) and $S^{2}$ (right) parameters for oxidized and reduced MIF. Gray shaded areas represent $\pm 1.5 \sigma$ from the linear least-squares fits of the data (black lines). (B) Per-residue differences in $R_{1} R_{2}$ (left) and $S^{2}$ (right) plotted as oxidized reduced. Bars in the shaded red area denote a greater value in oxMIF, while those in the blue area denote a greater value in reduced MIF. (C) Residues outside of the $1.5 \sigma$ correlation boundaries in (A) are mapped onto the MIF monomer (PDB: 1MIF), highlighting regions of the protein with redox-dependent differences in $\mu \mathrm{s}-\mathrm{ms}$ (left) and ps - ns (right) dynamics.

To confirm the influence of solution redox potential on $\mu$ s - ms dynamics in MIF, we quantified the exchange contribution to transverse relaxation, $R_{\mathrm{ex}}$, via CPMG relaxation dispersion experiments. The average global $R_{\text {ex }}$ value $\left(<R_{\mathrm{ex}}>\right)$ for MIF is fairly similar across the redox regimes tested, with local differences arising primarily under oxidizing conditions (Figure 3A). Numerous sites of $\mu$ s - ms dynamics are observed in all redox-modulated MIF samples (Figure S6), however, oxidizing solution stimulates the most widespread relaxation dispersion, affecting 39 residues ( $34 \%)$ in the MIF structure. These data are consistent with $R_{1} R_{2}$ values that estimated a stronger contribution from oxidizing solution to $\mu$ s - ms motions (Figure 2). 
125 Regions of MIF with known functional importance become flexible, including the monomer 126 interface (L26, I37, G50) and solvent channel allosteric site (196, Y99). Despite similar dynamics

127 in large portions of MIF, Figure 3B highlights selected sites that only appear flexible under 128 oxidizing conditions. With the exception of the solvent channel, these flexible sites have not been 129 well characterized for functional impact, suggesting that studies of MIF redox dependence can 130 reveal new target residues for structure-function correlations.
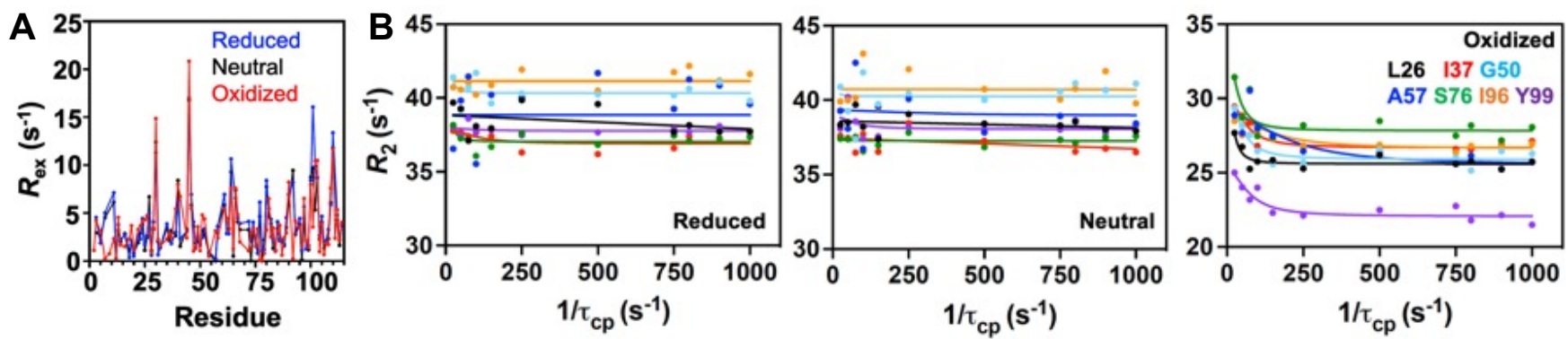

Figure 3. (A) Per-residue exchange contribution to relaxation ( $\left.R_{\mathrm{ex}}\right)$ for reduced (blue), redoxneutral (black), and oxidized MIF (red). (B) CPMG relaxation dispersion profiles for selected MIF residues collected in reducing (left), redox-neutral (center), or oxidizing (right) solution. These sites display evidence of $\mu \mathrm{s}-\mathrm{ms}$ flexibility under oxidizing conditions.

Cysteine 80 is a selectively modified redox switch

Prior work with a MIF epitope 22 suggested that C80 functions as a redox molecular switch.

140 However, redox sensitivity of C80 in the full-length trimeric protein is unknown. We performed

141 quantitative mass spectrometry to explore the possibility that C80 could be selectively modified

142 by redox reagents. Stepwise alkylation of MIF under each redox condition with iodoacetamide

143 (IAA) and N-ethylmaleimide (NEM) yields critical insight into the oxidation state of C80 in the

144 intact MIF trimer (Figures 4, S7, S8). Using NEM-bound C80 as a proxy for the abundance of

145 cystine residues reveals an increase from $75.1 \%$ to $91.3 \%$ cystine abundance between redox-

146 neutral and oxidizing conditions (Figure 4B). Under redox-neutral conditions, C80 exhibits the 
147 greatest variance in cystine abundance, further highlighting the reactivity and sensitivity of this

148 residue to environmental conditions. The relative abundance of cystine is also sensitive to the

149 amount of oxidative stress (Figure S8A). Increasing the concentration of oxidant in solution from

$150 \quad 1: 1$ to $2: 1$ shifts the C80 cystine relative abundance from $83.1 \%$ to $91.3 \%$. For comparison,

151 Figure S7B shows the expected contributions to the total ion intensity of C80 modified with IAA

152 or NEM if C80 is in a fully reduced, $50 \%$ reduced, or fully oxidized state. In addition to C80, MIF

153 also contains two cysteine residues in its ${ }^{56} \mathrm{CALC}{ }^{59}$ motif; however, the peptide fragment

154 containing C56 and C59 is a large 34-mer, precluding quantitation by the MS methods utilized

155 here. Although we focus on C80, we cannot rule out a significant role for other cysteines in MIF

156 redox dependence.
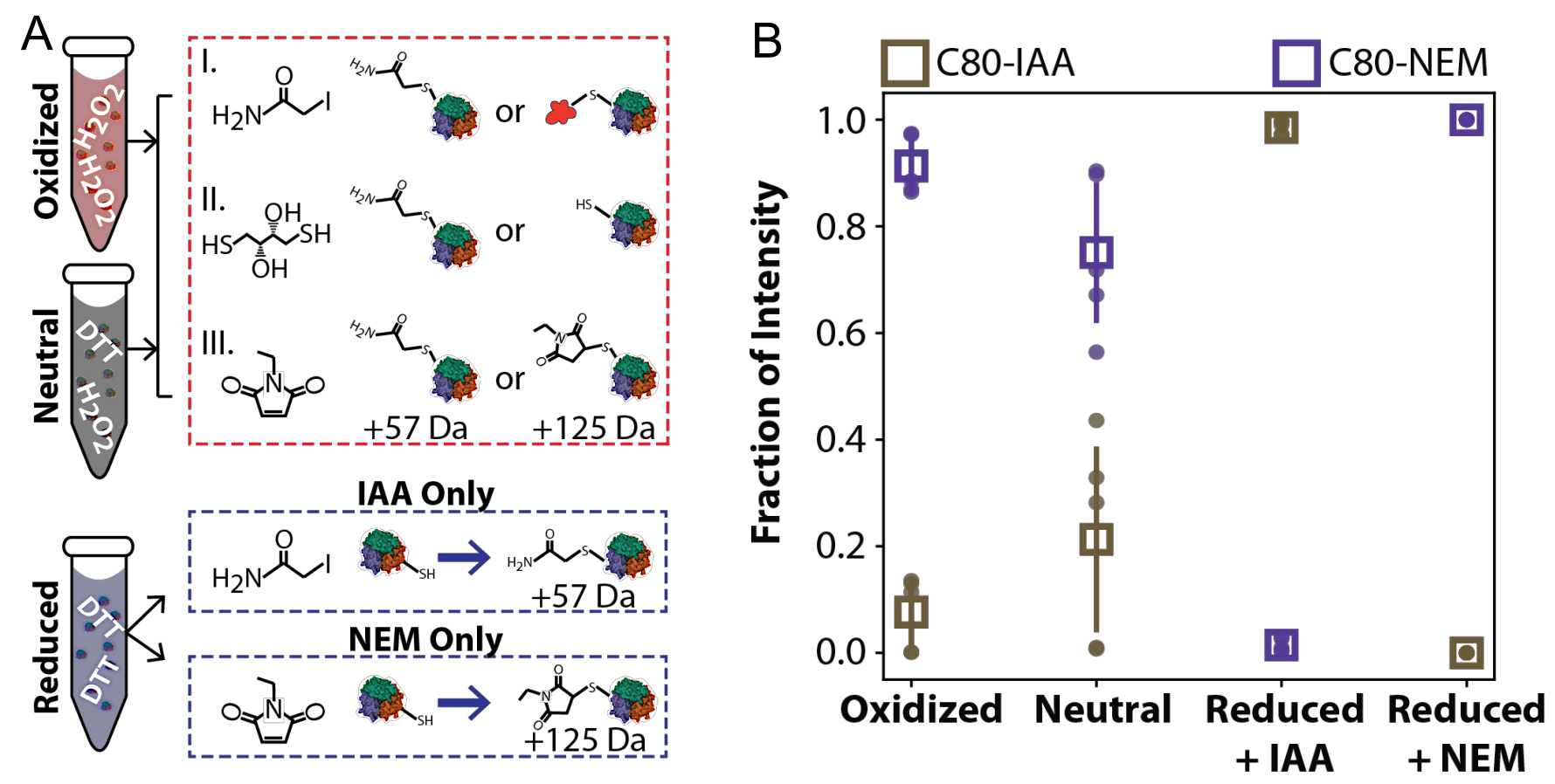

Figure 4. (A) Stepwise alkylation of MIF cysteine residues to determine the relative abundance of oxidized residues via mass spectrometry. The alkylation workflow involved three reaction steps (red, top panel). MIF equilibrated under reducing conditions was treated with only one alkylating agent and served as a quantitative control (blue, bottom panels). (B) Relative quantitation of $\mathrm{C} 80$ alkylation revealed conformational dependence and sensitivity to the 


\section{Mutation of redox-sensitive residues alters MIF structure and biological function}

To test the possibility that the redox sensitivity of MIF could reveal sites of functional impact that are not otherwise obvious, we created single point mutations at two residues displaying redox-dependent structural and/or dynamic changes, lysine 66 (K66) and cysteine 80 (C80). K66 was found to have a large variation in the $R_{1} R_{2}$ parameter in oxMIF, as well as a low ${ }^{1} \mathrm{H}-\left[{ }^{15} \mathrm{~N}\right]$ NOE in reduced MIF (Figures 2, S5), indicating that it undergoes significant changes in flexibility between the oxidized and reduced states. C80 also exhibits dynamic changes suggestive of redox-altered $\mu \mathrm{s}$ - ms chemical exchange and was shown by mass spectrometry to be selectively modified under oxidizing conditions (Figures 4, S5-8). ${ }^{1} \mathrm{H}^{15} \mathrm{~N}$ TROSY-HSQC NMR spectra of the K66A and C80A variants were collected in redox-neutral, reducing, and oxidizing solutions. Baseline structural changes caused by the $\mathrm{K} 66 \mathrm{~A}$ and $\mathrm{C} 80 \mathrm{~A}$ mutations are significant under redox-neutral conditions, particularly for the C80A variant, though CD spectroscopy indicates the variants are fully folded at NMR sample temperatures (Figures S9, S10). Interestingly, chemical shift perturbations observed for K66A MIF under oxidizing and reducing conditions are modest when compared to a redox-neutral K66A sample (Figures 5A, 5B). Most notably, oxidized K66A exhibits a chemical shift profile much smaller in magnitude than that observed in the corresponding wt-MIF experiments. In addition, reduced K66A exhibits larger chemical shift perturbations than oxidized in both number and magnitude, the inverse of what was found for wt-MIF. These data alone suggest that K66 may play a role in modulating the redox-dependent structural changes of wt-MIF, which are substantially diminished upon mutation. Likewise, the C80A variant strongly attenuates redox-sensitive NMR chemical shift changes in both reduced and oxMIF. These data, along with mass spectrometry, indicate that C80 modulates the conformational landscape of MIF under oxidative conditions. 

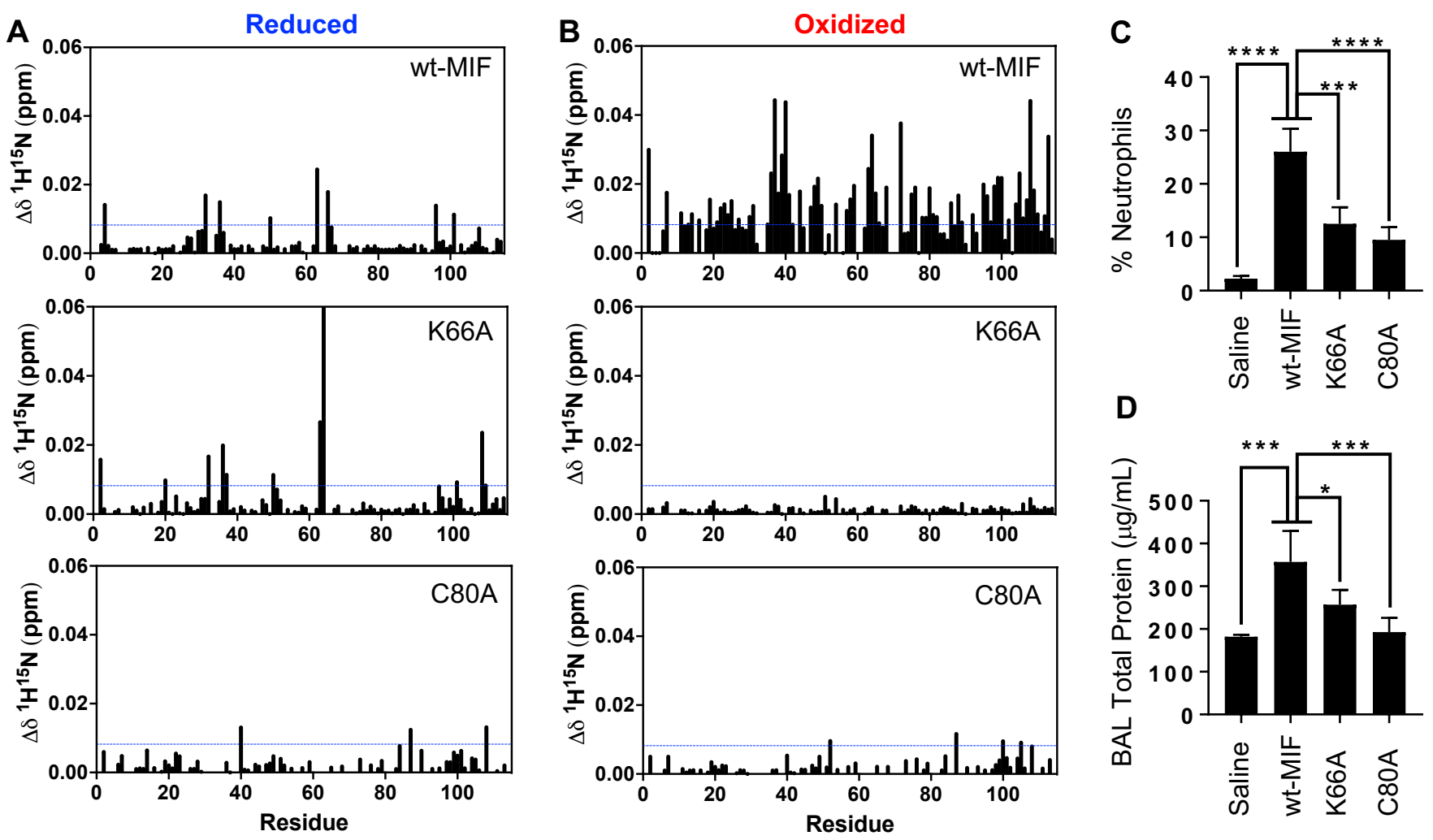

Figure 5. Mutation of the redox-sensitive K66 and C80 residues alters the redox-dependent MIF structure and hinders the ability of MIF to activate CD74 in vivo. K66A and C80A mutations alter the combined ${ }^{1} \mathrm{H}-{ }^{15} \mathrm{~N}$ chemical shift profiles of reduced $(A)$ and oxidized $(B) \mathrm{MIF}$, relative to redox-neutral K66A and $\mathrm{C} 80 \mathrm{~A}$ samples used as references. Blue lines denote $1.5 \sigma$ above the $10 \%$ trimmed mean of all shifts. Combined ${ }^{1} \mathrm{H}-{ }^{15} \mathrm{~N}$ chemical shift plots for reduced and oxidized wt-MIF are repeated here from Figure 1 for comparison. (C) K66A and C80A mutations significantly decreased CD74-dependent neutrophil recruitment in murine lungs in vivo. (D) Attenuated CD74-dependent neutrophil recruitment by K66A and C80A also led to significantly decreased pulmonary edema in vivo. ( $n=4$ in each group, ${ }^{*} p<0.05,{ }^{* * *} p \leq 0.001$ and $\left.{ }^{* * * *} p \leq 0.0001\right)$. Data are expressed as mean \pm SEM.

201 function, we evaluated the K66A and C80A variants in neutrophil recruitment assays in murine

202 lungs, where we measured total immune cells, percentage of neutrophils in BAL fluid, and protein

203 content (as a marker of alveolar capillary leak/pulmonary edema). This in vivo assay quantifies neutrophil recruitment on the surface of alveolar macrophages, an established metric for the 
205 MIF-induced activation of the pro-inflammatory CD74 receptor. ${ }^{34}$ The assay is indirect, using 206 chemokines released by activated macrophages to stimulate CXCR2 receptors on neutrophils 207 (that do not directly express CD74) to induce their migration to murine lungs. ${ }^{35}$ We found that 208 wt-MIF significantly increased the total BAL fluid cell counts over saline controls, while the K66A 209 and C80A variants showed a reduced number of total BAL fluid immune cells (Figure S11). The 210 neutrophil influx and pulmonary edema protein levels were significantly increased in the wt-MIF

211 group relative to vehicle controls (Figures 5C, 5D). In contrast, we observed significant

212 decreases in the groups to which the MIF variants were delivered, as compared to wt-MIF.

213 Together, our data indicate that the K66A and C80A variants have a functional effect in reducing 214 the inflammatory response of MIF in the murine lung. These findings also suggest that structural 215 and/or dynamic sensitivity of residues within MIF can provide a novel route for achieving 216 functional control beyond the known MIF active sites.

\section{Discussion}

We used solution redox potentials to modulate the MIF structure and dynamics in order to reveal changes in conformational equilibria and redox-sensitive residues that may be latent 220 functional sites within its structure. This work builds upon prior studies implying redox sensitivity 221 of MIF 11,22,36,37 as well as our recent paper highlighting a series of allosteric residues linking the 222 enzymatic (tautomerase) and CD74 binding sites of MIF. ${ }^{38}$ Here, we demonstrate that 223 examination of MIF redox sensitivity is another useful avenue for probing function beyond its 224 established active sites. Since MIF participates in both intracellular and extracellular 225 interactions, ${ }^{39}$ where the redox environments differ, understanding the implications of its redox 226 dependence may also inform its pro-inflammatory mechanisms and functional promiscuity, 227 which has been investigated at the biochemical, but not the structural, level. A previous 
228 characterization of the association of MIF with p53 tumor suppressor suggested the interaction

229 to be redox-dependent, having shown a significant decrease in coprecipitation in the presence

230 of a reductant. ${ }^{40}$ Further, interaction of MIF with ribosomal protein S-19 (RPS-19) was shown to 231 occur more strongly under reducing conditions. ${ }^{41}$ In addition, MIF is known to be post232 translationally modified by glycation in the brain tissue of Alzheimer's patients. Most notably, an 233 altered electrophoretic migratory behavior of glycated MIF was observed and demonstrated in 234 vitro by incubating MIF with glucose under oxidizing conditions. ${ }^{42}$

235 Redox signatures of inflammatory diseases, most of which implicate MIF, can also be 236 markedly different from those of healthy humans. The redox potential of epithelial lining fluid is 237110 to $-40 \mathrm{mV}$ in the lungs of asthmatics, which is significantly more oxidizing than that of healthy 238 humans (-150 to $-200 \mathrm{mV}) \cdot{ }^{27}$ Further, the synovial fluid of rheumatoid arthritis patients has been 239 shown to be highly oxidizing relative to that of a non-arthritic control group. ${ }^{43,44}$ A significant body 240 of literature suggests that when overexpressed at sites of inflammation, ${ }^{16}$ MIF can endure large 241 swings in environmental redox potential, consistent with our determination of the MIF redox 242 potential in vitro using solution $\mathrm{NMR}^{45,46}$ (Figure S12, midpoint $E_{0}^{\prime}=-215 \pm 5 \mathrm{mV}$, near normal 243 physiological range). NMR spin relaxation studies show redox-dependent modulation of local 244 flexibility in MIF, with motion observed on multiple timescales. oxMIF, in particular, exhibits the 245 most significant structural and dynamic changes, with widespread NMR spectral perturbations 246 and CPMG relaxation dispersion that show oxidizing potentials stimulate flexibility in $\sim 34 \%$ of 247 the MIF structure, including its redox-active residues. However, the majority of these flexible 248 regions have not been confirmed to harbor any functional impact in prior work.

It remains unclear how this relatively compact $(12.5 \mathrm{kDa}$ monomer) and symmetrical homotrimer accommodates a wide range of non-overlapping activities. A 2018 study showed 
251 that nanosecond dynamics were important for organization of the MIF-CD74 binding interface, ${ }^{6}$

252 and studies of a MIF epitope containing its ${ }^{56} \mathrm{CALC}^{59}$ motif showed selective binding to the Fab 253 fragment of the BaxB01 antibody under oxidizing conditions, after rearrangement of the Cys 254 sulfhydryls. ${ }^{22}$ Oxidizing conditions appear to promote a more flexible solvent channel, which we recently showed to be central to an allosteric mechanism regulating tautomerase and CD74 256 activation involving motion on multiple timescales. ${ }^{38}$ Given the number of MIF functions that have 257 yet to be explored at the molecular level and the fact that $\sim 100$ X-ray crystal structures of MIF 258 mutants, substrate-, and inhibitor-bound complexes have negligible variation in static structure 259 but vastly different NMR spectra, it is plausible, if not likely, that additional regions of functional 260 importance exist in MIF.

Here, we asked if changes in solution redox potential could reveal some of these latent

262 sites by modulating the structural and/or dynamic signatures of MIF. We experimentally altered 263 the redox potential of wt-MIF NMR samples, enabling us to identify redox-dependent structural 264 changes in residues that may be important for function. We mutated two such residues, K66 and 265 C80, that showed redox-sensitivity in wt-MIF and observed structural effects on MIF redox 266 behavior as well as altered functional responses. Having further confirmed the propensity of C80 267 for redox-driven modification by mass spectrometry, we speculate that MIF could act as a sensor 268 that toggles its conformation depending on its cellular environment to engage with varied binding 269 partners, as was previously suggested in a conformational control scheme for the ${ }^{56} \mathrm{CALC}^{59}$ 270 motif. The more extensive but partially-overlapping set of residues affected by the redox 271 environment suggests the regulatory network controlling non-overlapping MIF functions may be 272 larger than previously reported, where additional "allosteric nodes" may be revealed only when 273 MIF is exposed to stimuli, such as environmental factors associated with inflammation, serving 
274 as a natural means of activating latent residues to expand MIF functions under altered cellular

275 conditions. Speculating further, targeting small molecules to redox sensitive residues may 276 prevent MIF from engaging in certain downstream cascades. Future studies of protein-protein 277 interactions with supposed binding partners of MIF, such as thioredoxin ${ }^{47,48}$ or ribosomal protein $278 \mathrm{~S} 19,{ }^{41}$ as well as with small molecules, can evaluate this mechanism. Another interesting avenue 279 warranting further exploration is the redox-dependent interaction of MIF with its known inhibitors 280 or with novel compounds, as it presents an opportunity to preferentially target oxMIF, a form of 281 the protein that may appear predominately within the inflammatory state.

\section{Materials and Methods}

284 Protein expression, purification, and redox sample preparation

Wild-type (wt) and/or human MIF variants cloned into a pET11b vector were grown in 286 lysogeny broth (LB) for biochemical studies and in isotopically enriched M9 minimal medium 287 supplemented with $\mathrm{CaCl}_{2}, \mathrm{MgSO}_{4}$, and MEM vitamins for NMR, after adapting BL21(DE3) cells 288 to $\mathrm{D}_{2} \mathrm{O}$. Small cultures of MIF were grown overnight in $\mathrm{LB}$ at $37^{\circ} \mathrm{C}$ and used to inoculate cultures 289 containing $50 \% \mathrm{D}_{2} \mathrm{O}$ the following morning, which were grown $8-10$ hours at $37^{\circ} \mathrm{C}$ and then 290 used to inoculate cultures containing $95 \% \mathrm{D}_{2} \mathrm{O}$, which were incubated at $37^{\circ} \mathrm{C}$ for 12 hours. The 291 cells were collected by centrifugation and resuspended in M9 medium ( $\left.1 \mathrm{~L}, 100 \% \mathrm{D}_{2} \mathrm{O}\right)$ 292 supplemented with ${ }^{15} \mathrm{NH}_{4} \mathrm{Cl}$ (Cambridge Isotope Laboratories) and ${ }^{12} \mathrm{C}_{6} \mathrm{H}_{12} \mathrm{O}_{6}$ as the sole 293 nitrogen and carbon sources, respectively. Cultures were grown to an $\mathrm{OD}_{600}$ of $0.8-1.0$ and 294 induced with $1 \mathrm{mM}$ isopropyl $\beta$-D-1-thiogalactopyranoside (IPTG). Following 16-18 hours of post295 induction growth, the cells were harvested by centrifugation. 
The cells were resuspended in $\sim 30 \mathrm{~mL}$ of an ice-cold buffer of $20 \mathrm{mM}$ Tris- $\mathrm{HCl}, 20 \mathrm{mM}$

$297 \mathrm{NaCl}$, and $1 \mathrm{mM}$ EDTA at pH 7.4 supplemented with protease inhibitors, lysed by ultrasonication,

298 clarified by centrifugation, filtered, and purified with a $120 \mathrm{~mL}$ Macro-Prep High Q

299 column(BioRad) pre-equilibrated with the lysis buffer. MIF does not bind the Q resin and is found

300 in the flow-through. MIF was purified to its final form on a HiLoad 26/600 Superdex 200 size

301 exclusion column (GE Healthcare). For NMR samples, MIF was dialyzed into a buffer containing

$30220 \mathrm{mM}$ sodium phosphate, $1 \mathrm{mM}$ EDTA, and 7.5\% $\mathrm{D}_{2} \mathrm{O}$ at $\mathrm{pH}$ 7.0. Final concentrations of MIF

303 were $0.5-1 \mathrm{mM}$ determined using $\varepsilon_{280}=12,950 \mathrm{M}^{-1} \mathrm{~cm}^{-1} .49$

304 Oxidation of MIF was achieved by addition of $\mathrm{H}_{2} \mathrm{O}_{2}$ to a final concentration of $6 \mathrm{mM}$ in an

305 aliquot of $0.5-1 \mathrm{mM}$ MIF. Reduced MIF was prepared with a $6 \mathrm{mM}$ final concentration of DTT.

306 Redox-neutral samples were prepared with equimolar amounts of $\mathrm{DTT} / \mathrm{H}_{2} \mathrm{O}_{2}$. Stocks of the

307 oxidizing and reducing agents were freshly prepared in a degassed buffer of 20 mM sodium

308 phosphate, $1 \mathrm{mM}$ EDTA, and 7.5\% $\mathrm{D}_{2} \mathrm{O}$ at $\mathrm{pH}$ 7.0. Following addition of oxidant or reductant to

309 MIF samples, the NMR tubes were briefly degassed with nitrogen and sealed with Parafilm

310 (Bemis). The samples were equilibrated in the presence of redox reagents for at least six hours.

311 Circular Dichroism (CD) Spectroscopy

312 CD spectra and thermal unfolding experiments were recorded on a JASCO J-815

313 spectropolarimeter equipped with a variable temperature Peltier device. Denaturation curves of

$31410 \mu \mathrm{M}$ MIF were recorded at $218 \mathrm{~nm}$ in a $2 \mathrm{~mm}$ quartz cuvette. The temperature range for each

315 scan was $20-100{ }^{\circ} \mathrm{C}(293 \mathrm{~K}-373 \mathrm{~K})$. Thermodynamic parameters were extracted via nonlinear

316 curve fitting of CD data to Equation 1 in GraphPad Prism:

317 Ellipticity $(T)=\frac{\left[\left(m_{f} T+b_{f}\right)+\left(m_{u} T+b_{u}\right)\right] \exp \left[\left(-\frac{\Delta H_{D, v H}}{R}\right)\left(\frac{1}{T}-\frac{1}{T_{m}}\right)\right]}{1+\exp \left[\left(-\frac{\Delta H_{D, v H}}{R}\right)\left(\frac{1}{T}-\frac{1}{T_{m}}\right)\right]}$ 
318 where $m_{\mathrm{f}}, b_{\mathrm{f}}$, and $m_{\mathrm{u}}, b_{\mathrm{u}}$ are the slopes and y-intercepts of the folded (low temperature) and

319 unfolded (high temperature) regions of the melting curve, $R$ is the gas constant, $T_{\mathrm{m}}$ and $\Delta H_{\mathrm{D}, \mathrm{vH}}$ 320 are the unfolding midpoint and van't Hoff enthalpy of denaturation at $T_{\mathrm{m}}$, respectively. Free 321 energy analysis was performed as described elsewhere, ${ }^{50}$ using values of $\Delta C_{p}$, the unfolding 322 heat capacity, estimated from the report of Privalov. ${ }^{51,52}$ The differences in apparent unfolding 323 enthalpies, entropies, and free energies at a reference temperature ( $T_{\mathrm{REF}}$ ) of $298 \mathrm{~K}$ were 324 calculated as;

$325 \Delta H_{D, a p p}=\Delta H_{D, v H}+\Delta C_{p}\left(T_{R E F}-T_{m}\right)$

$\Delta S_{D, a p p}=\frac{\Delta H_{D, v H}}{T_{m}}+\Delta C_{p} \ln \left(\frac{T_{R E F}}{T_{m}}\right)$

$\Delta G(T)=\Delta H_{D, v H}\left(1-\frac{T_{R E F}}{T_{m}}\right)+\Delta C_{p}\left[\left(T_{R E F}-T_{m}\right)-T_{R E F} \ln \left(\frac{T_{R E F}}{T_{m}}\right)\right]$

328 These data are reported as "apparent" values due to the $\sim 90 \%$ reversibility of the MIF unfolding 329 transition.

NMR Spectroscopy

NMR experiments were performed on a Bruker Avance NEO $600 \mathrm{MHz}$ or Bruker

Avance III HD $850 \mathrm{MHz}$ spectrometer at $30^{\circ} \mathrm{C}$. NMR data were processed using NMRPipe ${ }^{53}$

333 and analyzed in Sparky ${ }^{54}$ along with in-house scripts. The $\mathrm{pH}$ of NMR samples was monitored

334 after addition of redox reagents to ensure that NMR spectral changes were due to

335 reduction/oxidation. NMR assignments for wt-MIF and the K66A and C80A variants were

336 confirmed from standard triple resonance experiments. Combined chemical shift perturbations

$337(\Delta \delta)$ were determined by $\Delta \delta=\sqrt{\left(\delta_{H N}^{2}\right)+\left(\delta_{N}^{2} / 25\right)}$. The HSQC of oxidized MIF was processed

338 with an exponential window function in both the direct and indirect dimensions in

339 NMRPipe. The peak intensity and volume for each pair of slow exchanging peaks were 
340 calculated in SPARKY, where peak volume was calculated by integrating each peak as a

341 Lorentzian. The minor state populations were estimated by taking the volume of the minor

342 peak and dividing by the total volume of the slow exchanging pairs.

NMR spin relaxation experiments were performed using TROSY-based pulse

344 sequences adapted from Palmer and coworkers. ${ }^{55}$ Relaxation data were acquired with the ${ }^{1} \mathrm{H}$

345 and ${ }^{15} \mathrm{~N}$ carriers set to the water resonance and $120 \mathrm{ppm}$, respectively. Longitudinal relaxation

346 rates $\left(R_{1}\right)$ were measured with $T_{1}$ delays of $0,20,60,100,200,600,800$, and $1200 \mathrm{~ms}$.

347 Transverse relaxation rates $\left(R_{2}\right)$ were collected with 1.0 ms spacing between $180^{\circ} \mathrm{CPMG}$

348 pulses at total relaxation delays of $0,1,2,4,8$, and 10 , and $12 \mathrm{~ms}$. The recycle delay in these

349 experiments was 2.5 s. Longitudinal and transverse relaxation rates were extracted by non-

350 linear least squares fitting of the peak heights (major peaks in cases of slow exchange) to a

351 single exponential decay using in-house software. Uncertainties in these rates were

352 determined from replicate spectra. The heteronuclear cross-relaxation rate $\left({ }^{1} \mathrm{H}-\left[{ }^{15} \mathrm{~N}\right] \mathrm{NOE}\right)$ was

353 obtained by interleaving pulse sequences with and without proton saturation and calculated

354 from the ratio of peak heights from these experiments. Carr-Purcell-Meiboom-Gill (CPMG)

355 NMR experiments were performed at $30^{\circ} \mathrm{C}$ with a constant relaxation period of $40 \mathrm{~ms}$, a $2.0 \mathrm{~s}$

356 recycle delay, and $\tau_{\mathrm{cp}}$ points of $0,25,50,75,100,150,250,500,750,800,900$, and $1000 \mathrm{~ms}$.

357 Relaxation dispersion profiles were generated by plotting $R_{2}$ vs. $1 / \tau_{\mathrm{cp}}$ and exchange

358 parameters were obtained from fits of these data carried out with in-house scripts.

359 Uncertainties were obtained from replicate spectra.

$360 \quad$ NMR spin-relaxation rates were fit to one of five semi-empirical forms of the spectral

361 density function using model-free analysis. ${ }^{56,57}$ Fitting of motional parameters was performed in

362 RELAX. ${ }^{58,59}$ The criteria for inclusion of residues in the diffusion tensor estimate relied on the 
363 method of Tjandra and coworkers. ${ }^{47} \mathrm{~N}-\mathrm{H}$ bond lengths were assumed to be $1.02 \AA$ and the ${ }^{15} \mathrm{~N}$

364 chemical shift anisotropy was set to -160 ppm. During the model selection process, the diffusion

365 tensor parameters were optimized simultaneously, and model selection was repeated until the 366 optimized tensor parameters and order parameter $\left(S^{2}\right)$ did not differ from those of the previous 367 iteration.

368 Mass Spectrometry

369 Cysteine and cystine residues of purified MIF equilibrated under each of the redox 370 conditions were alkylated in a stepwise process with iodoacetamide (IAA) followed by $N$ 371 ethylmaleimide (NEM). Parallel reaction monitoring-mass spectrometry captured the oxidation 372 states of C80 under different redox conditions. Skyline software ${ }^{60}$ was used to calculate the 373 relative abundance of cysteine and cystine at the C80 position, indicated by binding to IAA or 374 NEM. Additional methodological details can be found in the Supporting Information.

375 In Vivo Neutrophil Recruitment Assay

All animal studies were approved by the Institutional Animal Care and Use Committee of

377 Cooper University Healthcare, Camden, NJ. The wt mice of genetic background strain 378 (C57BL6/J) were purchased from the Jackson Laboratory (Bar Harbor, ME). Mice were housed 379 at the pathogen-free animal facility at Cooper University Healthcare. All experiments were done 380 in 9-10 week old male mice. Mice were administered a one-time intratracheal instillation of 100 $381 \mu \mathrm{l}$ of normal saline alone (vehicle) or normal saline solution containing $1 \mu \mathrm{g}$ of wt-MIF, K66A MIF 382 or C80A MIF. These mice were sacrificed after $6 \mathrm{~h}$ of vehicle only or vehicle + experimental 383 agent administration. Bronchoalveolar lavage (BAL) was performed by cannulating the trachea 384 with a blunt 22-gauge needle and lavaging both lungs with $800 \mu$ of sterile PBS solution. 385 Bronchoalveolar lavage fluid (BALF) was harvested, and total cell counts in BALF were 
determined using the TC20 automated cell counter (Bio-Rad Laboratories, Inc., Hercules, CA).

387 The differential cell counts were performed on cells cytocentrifuged onto glass slides (Fisher 388 Scientific) stained with the Hema 3 Staining System (Fisher Diagnostics, Middletown, VA) and 389 cell differential was tabulated using light microscopy. Total protein concentration in the BAL fluid 390 was measured using the Pierce ${ }^{\mathrm{TM}}$ BCA assay kit (Thermo Scientific, Rockford, IL), as previously 391 described. 6

\section{Acknowledgments}

394 The authors thank J. Patrick Loria for helpful discussions. This work was supported by Rhode Island Foundation Grant GR5290658 and funds from the Office of the Vice President for

Research at Brown University (to GPL).

\section{Competing Interests}

399 The authors declare no competing interests.

\section{References}

(1) Bernhagen, J. r.; Bucala, R.: MIF Family Cytokines in Innate Immunity and Homeostasis. In Progress in Inflammation Research,; 1st ed,; Springer International Publishing : Imprint: Springer,: Cham, 2017.

(2) Leng, L.; Metz, C. N.; Fang, Y.; Xu, J.; Donnelly, S.; Baugh, J.; Delohery, T.; Chen, Y.; Mitchell, R. A.; Bucala, R. MIF signal transduction initiated by binding to CD74. J Exp Med 2003, 197, 1467-1476.

(3) Bernhagen, J.; Krohn, R.; Lue, H.; Gregory, J. L.; Zernecke, A.; Koenen, R. R.; Dewor, M.; Georgiev, I.; Schober, A.; Leng, L.; Kooistra, T.; Fingerle-Rowson, G.; Ghezzi, P.; Kleemann, R.; McColl, S. R.; Bucala, R.; Hickey, M. J.; Weber, C. MIF is a noncognate ligand of CXC chemokine receptors in inflammatory and atherogenic cell recruitment. Nat Med 2007, 13, 587-596.

(4) Meyer-Siegler, K. L.; Iczkowski, K. A.; Leng, L.; Bucala, R.; Vera, P. L. Inhibiton of Macrophage Migration Inhibitory Factor or its Receptor (CD74) Attenuates Growth and Invasion of DU145 Prostate Cancer Cells. J. Immunol. 2006, 177, 8730-8739. 
(5) Cournia, Z.; Leng, L.; Gandavadi, S.; Du, X.; Bucala, R.; Jorgensen, W. L. Discovery of Human Macrophage Migration Inhibitory Factor (MIF)-CD74 Amtagonists via Virtual Screening. J. Med. Chem. 2009, 52, 416-424.

(6) Pantouris, G.; Ho, J.; Shah, D.; Syed, M. A.; Leng, L.; Bhandari, V.; Bucala, R.; Batista, V. S.; Loria, J. P.; Lolis, E. J. Nanosecond Dynamics Regulate the MIF-induced Activity of CD74. Angew. Chem. Int. Ed. 2018, 57, 7116-7119.

(7) Mizue, Y.; Ghani, S.; Leng, L.; McDonald, C.; Kong, P.; Baugh, J.; Lane, S. J.; Craft, J.; Nishihira, J.; Donnelly, S. C.; Zhu, Z.; Bucala, R. Role for Macrophage Migration Inhibitory Factor in Asthma. Proc. Natl. Acad. Sci. USA 2005, 102, 14410-14415.

(8) Joshi, P. C.; Poole, G. V.; Sachdev, V.; Zhou, X.; Jones, Q. Trauma Patients with Positive Cultures have Higher Levels of Circulating Macrophage Migration Inhibitory Factor. Res. Commun. Mol. Pathol. Pharmacol. 2000, 107, 13-20.

(9) Kleeman, R.; Hausser, A.; Geiger, G.; Mischke, R.; Burger-Kentischer, A.; Flieger, O.; Johannes, F. J.; Roger, T.; Calandra, T.; Kapurniotu, A.; Grell, M.; Finkelmeier, D.; Brunner, H.; Bernhagen, J. Intracellular Action of the Cytokine MIF to Modulate AP-1 Activity and the Cell Cycle Through JAB-1. Nature 2000, 408, 211-216.

(10) Calandra, T.; Bernhagen, J.; Metz, C. N.; Spiegel, L. A.; Bacher, M.; Donnelly, T.; Cerami, A.; Bucala, R. MIF as a Glucocorticoid-Induced Modulator of Cytokine Production. Nature 1995, 377, 68-71.

(11) Thiele, M.; Bernhagen, J. Link between Macrophage Migration Inhibitory Factor and Cellular Redox Regulation. Antioxid. Redox Signal. 2005, 7, 1234-1248.

(12) Morand, E. F.; Leech, M.; Bernhagen, J. MIF: A New Cytokine Link between Rheumatoid Arthritis and Atherosclerosis. Nat. Rev. Drug Discov. 2006, 5, 399-410.

(13) de Jong, Y. P.; Abadia-Molina, A. C.; Satoskar, A. R.; Clarke, K.; Rietdijk, S. T.; Faubion, W. A.; Mizoguchi, E.; Metz, C. N.; Alsahli, M.; ten Hove, T.; Keates, A. C.; Lubetsky, J. B.; Farrell, R. J.; Michetti, P.; van Deventer, S. J.; Lolis, E.; David, J. R.; Bhan, A. K.; Terhorst, C. Development of Chronic Colitis is Dependent on the Cytokine MIF. Nat. Immunol. 2001, 2, 1061-1066.

(14) Makhija, R.; Kingsnorth, A.; Demaine, A. Gene Polymorphisms of the Macrophage Migration Inhibitory Factor and Acute Pancreatitis. J.Pancreas 2007, 8, 289-295.

(15) Donnelly, S. C.; Haslett, C.; Reid, P. T.; Grant, I. S.; Wallace, W. A.; Metz, C. N.; Bruce, L. J.; Bucala, R. Regulatory Role for Macrophage Migration Inhibitory Factor in Acute Respiratory Distress Syndrome. Nat. Med. 1997, 3, 320-323.

(16) Bucala, R.; Donnelly, S. C. Macrophage Migration Inhibitory Factor: A Probable Link between Inflammation and Cancer. Immunity 2007, 26, 281-285.

(17) Balogh, K. N.; Templeton, D. J.; Cross, J. V. Macrophage Migration Inhibitory Factor Protects Cancer Cells from Immunogenic Cell Death and Impairs Anti-tumor Responses. PLOS One 2018, 13, e0197702.

(18) Richard, V.; Kindt, N.; Saussez, S. Macrophage Migration Inhibitory Factor Involvement in Breast Cancer. Int. J. Oncol. 2015, 47, 1627-1633.

(19) Agarwal, R.; Whang, D. H.; Alvero, A. B.; Visintin, I.; Lai, Y. L.; Segal, E. A.; Schwartz, P.; Ward, D.; Rutherford, T.; Mor, G. Macrophage Migration Inhibitory Factor Expression in Ovarian Cancer. Am. J. Obstet. Gynecol. 2007, 196, 348.e341-348.e345.

(20) Nobre, C. C. G.; de Arajuo, J. M. G.; Fernandes, T. A. A. M.; Cobucci, R. N. O.; Lanza, D. C. F.; Andrade, V. S.; Fernandes, J. V. Macrophage Migration Inhibitory Factor (MIF): Biological Activities and Relation with Cancer. Pathol. Oncol. Res. 2017, 23, 235-244. 
(21) Bleilevens, C.; Soppert, J.; Hoffmann, A.; Breuer, T.; Bernhagen, J.; Martin, L.; Stiehler, L.; Marx, G.; Dreher, M.; Stoppe, C.; Simon, T. P. Macrophage Migration Inhibitory Factor (MIF) Plasma Concentration in Critically Ill COVID-19 Patients: A Prospective Observational Study. Diagnostics (Basel) 2021, 11 .

(22) Schinagl, A.; Kerschbaumer, R. J.; Sabarth, N.; Douillard, P.; Scholz, P.; Voelkel, D.; Hollerweger, J. C.; Goettig, P.; Brandstetter, H.; Scheiflinger, F.; Thiele, M. Role of the Cysteine 81 Residue of Macrophage Migration Inhibitory Factor as a Molecular Redox Switch. Biochemistry 2018, 57, 1523-1532.

(23) Kleemann, R.; Kapurniotu, A.; Frank, R. W.; Gessner, A.; Mischke, R.; Flieger, O.; Juttner, S.; Brunner, H.; Bernhagen, J. Disulfide analysis reveals a role for macrophage migration inhibitory factor (MIF) as thiol-protein oxidoreductase. J Mol Biol 1998, 280, 85-102.

(24) Kleemann, R.; Mischke, R.; Kapurniotu, A.; Brunner, H.; Bernhagen, J. Specific reduction of insulin disulfides by macrophage migration inhibitory factor (MIF) with glutathione and dihydrolipoamide: potential role in cellular redox processes. FEBS Lett 1998, 430, 191-196.

(25) Mittal, M.; Siddiqui, M. R.; Tran, K.; Reddy, S. P.; Malik, A. B. Reactive oxygen species in inflammation and tissue injury. Antioxid Redox Signal 2014, 20, 1126-1167.

(26) Fahy, J. V.; Corry, D. B.; Boushey, H. A. Airway Inflammation and Remodeling in Asthma. Curr. Opin. Pulm. Med. 2000, 6, 15-20.

(27) Fitzpatrick, A. M.; Teague, W. G.; Holguin, F.; Yeh, M.; Brown, L. A. Airway Glutathione Homeostasis is Altered in Children with Severe Asthma: Evidence for Oxidant Stress. J. Allergy Clin. Immunol. 2009, 123, 146-152.

(28) Watson, W.; Ritzenthaler, J. D.; Roman, J. Lung Extracellular Matrix and Redox Regulation. Redox Biol. 2016, 8, 303-315.

(29) Kinnula, V. L.; Fattman, C. L.; Tan, R. J.; Oury, T. D. Oxidative Stress in Pulmonary Fibrosis: A Possible Role for Redox Modulatory Therapy. Am. J. Respir. Crit. Care Med. 2005, 172, 417422.

(30) Bakalova, R.; Zhelev, Z.; Aoki, I.; Saga, T. Tissue Redox Activity as a Hallmark of Carcinogenesis: From Early to Terminal Stages of Cancer. Clin. Cancer Res. 2013, 19, 2503-2517.

(31) Kneller, J. M.; Lu, M.; Bracken, C. An Effective Method for the Discrimination of Motional Anisotropy and Chemical Exchange. J. Am. Chem. Soc. 2002, 124, 1852-1853.

(32) Pantouris, G.; Syed, M. A.; Fan, C.; Rajasekaran, D.; Cho, T. Y.; Rosenberg, E. M., Jr.; Bucala, R.; Bhandari, V.; Lolis, E. J. An Analysis of MIF Structural Features that Control Functional Activation of CD74. Chem Biol 2015, 22, 1197-1205.

(33) Lubetsky, J. B.; Dios, A.; Han, J.; Aljabari, B.; Ruzsicska, B.; Mitchell, R.; Lolis, E.; AlAbed, Y. The tautomerase active site of macrophage migration inhibitory factor is a potential target for discovery of novel anti-inflammatory agents. J Biol Chem 2002, 277, 24976-24982.

(34) Takahashi, K.; Koga, K.; Linge, H. M.; Zhang, Y.; Lin, X.; Metz, C. N.; Al-Abed, Y.; Ojamaa, K.; Miller, E. J. Macrophage CD74 contributes to MIF-induced pulmonary inflammation. Respir Res 2009, 10, 33.

(35) Mehrad, B.; Strieter, R. M.; Moore, T. A.; Tsai, W. C.; Lira, S. A.; Standiford, T. J. CXC chemokine receptor-2 ligands are necessary components of neutrophil-mediated host defense in invasive pulmonary aspergillosis. J Immunol 1999, 163, 6086-6094.

(36) Nguyen, M. T.; Beck, J.; Lue, H.; Funfzig, H.; Kleemann, R.; Koolwijk, P.; Kapurniotu, A.; Bernhagen, J. A 16-residue peptide fragment of macrophage migration inhibitory factor, MIF-(50-65), exhibits redox activity and has MIF-like biological functions. J Biol Chem 2003, 278, 33654-33671. 
(37) Takahashi, M.; Nishihira, J.; Shimpo, M.; Mizue, Y.; Ueno, S.; Mano, H.; Kobayashi, E.; Ikeda, U.; Shimada, K. Macrophage migration inhibitory factor as a redox-sensitive cytokine in cardiac myocytes. Cardiovasc Res 2001, 52, 438-445.

(38) Pantouris, G.; Khurana, L.; Ma, A.; Skeens, E.; Reiss, K.; Batista, V. S.; Lisi, G. P.; Lolis, E. J. Regulation of MIF Enzymatic Activity by an Allosteric Site at the Central Solvent Channel. Cell Chem Biol 2020, 27, 740-750 e745.

(39) Calandra, T.; Roger, T. Macrophage migration inhibitory factor: a regulator of innate immunity. Nat Rev Immunol 2003, 3, 791-800.

(40) Jung, H.; Seong, H. A.; Ha, H. Critical role of cysteine residue 81 of macrophage migration inhibitory factor (MIF) in MIF-induced inhibition of p53 activity. J Biol Chem 2008, 283, 20383-20396.

(41) Filip, A. M.; Klug, J.; Cayli, S.; Frohlich, S.; Henke, T.; Lacher, P.; Eickhoff, R.; Bulau, P.; Linder, M.; Carlsson-Skwirut, C.; Leng, L.; Bucala, R.; Kraemer, S.; Bernhagen, J.; Meinhardt, A. Ribosomal protein S19 interacts with macrophage migration inhibitory factor and attenuates its proinflammatory function. J Biol Chem 2009, 284, 7977-7985.

(42) Kassaar, O.; Pereira Morais, M.; Xu, S.; Adam, E. L.; Chamberlain, R. C.; Jenkins, B.; James, T. D.; Francis, P. T.; Ward, S.; Williams, R. J.; van den Elsen, J. Macrophage Migration Inhibitory Factor is subjected to glucose modification and oxidation in Alzheimer's Disease. Sci Rep 2017, 7, 42874.

(43) Maurice, M. M.; Nakamura, H.; van der Voort, E. A.; van Vliet, A. I.; Staal, F. J.; Tak, P. P.; Breedveld, F. C.; Verweij, C. L. Evidence for the role of an altered redox state in hyporesponsiveness of synovial T cells in rheumatoid arthritis. J Immunol 1997, 158, 1458-1465.

(44) Wu, S. M.; Pizzo, S. V. alpha(2)-Macroglobulin from rheumatoid arthritis synovial fluid: functional analysis defines a role for oxidation in inflammation. Arch Biochem Biophys 2001, 391, 119126.

(45) Taylor, S. L.; Crawley-Snowdon, H.; Wagstaff, J. L.; Rowe, M. L.; Shepherd, M.; Williamson, R. A.; Howard, M. J. Measuring protein reduction potentials using 15N HSQC NMR spectroscopy. Chem Commun (Camb) 2013, 49, 1847-1849.

(46) Zimmermann, J.; Kuhne, R.; Sylvester, M.; Freund, C. Redox-regulated conformational changes in an SH3 domain. Biochemistry 2007, 46, 6971-6977.

(47) Tjandra, N.; Feller, S. E.; Pastor, R. W.; Bax, A. Rotational diffusion anisotropy of human ubiquitin from N-15 NMR relaxation. J Am Chem Soc 1995, 117, 12562-12566.

(48) Son, A.; Kato, N.; Horibe, T.; Matsuo, Y.; Mochizuki, M.; Mitsui, A.; Kawakami, K.; Nakamura, H.; Yodoi, J. Direct Association of Thioredoxin-1 (TRX) with Macrophage Migration Inhibitory Factor (MIF): Regulatory Role of TRX on MIF Internalization and Signaling Antioxid. Redox Signal. 2009, 11, 2595-2605.

(49) Lubetsky, J. B.; Swope, M.; Dealwis, C.; Blake, P.; Lolis, E. Pro-1 of macrophage migration inhibitory factor functions as a catalytic base in the phenylpyruvate tautomerase activity. Biochemistry 1999, 38, 7346-7354.

(50) Zhong, F.; Lisi, G. P.; Collins, D. P.; Dawson, J. H.; Pletneva, E. V. Redox-dependent stability, protonation, and reactivity of cysteine-bound heme proteins. Proc Natl Acad Sci US A 2014, 111, E306-315.

(51) Makhatadze, G. I.; Privalov, P. L. Heat capacity of proteins. I. Partial molar heat capacity of individual amino acid residues in aqueous solution: hydration effect. J Mol Biol 1990, 213, 375-384.

(52) Privalov, P. L.; Makhatadze, G. I. Contribution of hydration and non-covalent interactions to the heat capacity effect on protein unfolding. J Mol Biol 1992, 224, 715-723.

(53) Delaglio, F.; Grzesiek, S.; Vuister, G. W.; Zhu, G.; Pfeifer, J.; Bax, A. NMRPipe: a multidimensional spectral processing system based on UNIX pipes. J Biomol NMR 1995, 6, 277-293. 
(54) Lee, W.; Tonelli, M.; Markley, J. L. NMRFAM-SPARKY: enhanced software for biomolecular NMR spectroscopy. Bioinformatics 2015, 31, 1325-1327.

(55) Loria, J. P.; Rance, M.; Palmer, A. G. A relaxation-compensated Carr-Purcell-MeiboomGill sequence for characterizing chemical exchange by NMR spectroscopy. J Am Chem Soc 1999, 121, 2331-2332.

(56) Clore, G. M.; Szabo, A.; Bax, A.; Kay, L. E.; Driscoll, P. C.; Gronenborn, A. M. Deviations from the Simple 2-Parameter Model-Free Approach to the Interpretation of N-15 Nuclear MagneticRelaxation of Proteins. J Am Chem Soc 1990, 112, 4989-4991.

(57) Lipari, G.; Szabo, A. Model-Free Approach to the Interpretation of Nuclear MagneticResonance Relaxation in Macromolecules .2. Analysis of Experimental Results. J Am Chem Soc 1982, $104,4559-4570$.

(58) d'Auvergne, E. J.; Gooley, P. R. Optimisation of NMR dynamic models I. Minimisation algorithms and their performance within the model-free and Brownian rotational diffusion spaces. $J$ Biomol NMR 2008, 40, 107-119.

(59) d'Auvergne, E. J.; Gooley, P. R. Optimisation of NMR dynamic models II. A new methodology for the dual optimisation of the model-free parameters and the Brownian rotational diffusion tensor. J Biomol NMR 2008, 40, 121-133.

(60) MacLean, B.; Tomazela, D. M.; Shulman, N.; Chambers, M.; Finney, G. L.; Frewen, B.; Kern, R.; Tabb, D. L.; Liebler, D. C.; MacCoss, M. J. Skyline: an open source document editor for creating and analyzing targeted proteomics experiments. Bioinformatics 2010, 26, 966-968. 